\title{
Subpallial DIx2-Expressing Cells Give Rise to Astrocytes and Oligodendrocytes in the Cerebral Cortex and White Matter
}

\author{
Christine A. G. Marshall ${ }^{1}$ and James E. Goldman ${ }^{1,2}$ \\ ${ }^{1}$ Center for Neurobiology and Behavior, and 2Division of Neuropathology, Department of Pathology, Columbia University, \\ College of Physicians and Surgeons, New York, New York 10032
}

The precise origins of postnatal subventricular zone (SVZ) cells are not known. Furthermore, the gliogenic potential of progenitors expressing $D / x$ genes that migrate ventrodorsally from the ganglionic eminences has not been explored in vivo. Here, we identify the embryonic origins of two distinct populations of postnatal SVZ cells: SVZ border cells, which express Zebrin II, and migratory cells in the central SVZ, which are generally devoid of Zebrin II expression (Staugaitis et al., 2001). Zebrin II is expressed by all cells of the telencephalic primordium, with its expression becoming restricted to astrocytes in the mature telencephalon. As the neuroepithelium folds during corticostriatal sulcus formation (embryonic day 13-15), a wedge of Zebrin II + cells is created at the presumptive site of the dorsolateral SVZ. At this time, Dlx2-expressing cells and their progeny begin to migrate ventrodorsally along a medial path from the ganglionic eminences. These migratory subpallial cells invade the

Astrocytes and oligodendrocytes are generated throughout embryonic and postnatal development in the murine telencephalon. Astrocytes originate from two sources: from the neuroepithelium, via a radial glial phenotype (Luskin et al., 1988; Price and Thurlow, 1988; Voigt, 1989), and from migratory progenitors that emerge from the dorsolateral subventricular zone (SVZ) to colonize adjacent gray and white matter (Levison and Goldman, 1993; Luskin and McDermott, 1994). Oligodendrocytes are thought to originate from the ventral neuroepithelium and to become specified by exposure to Sonic hedgehog (Shh) during embryogenesis (Woodruff et al., 2001). However, it is not clear whether all oligodendrocytes are specified ventrally. Postnatally, progenitors emigrate from the SVZ into the striatum, white matter, and cortex, where some develop into oligodendrocytes (Levison and Goldman, 1993; Luskin and McDermott, 1994). Many of these cells are not committed irrevocably to an oligodendrocyte fate, as SVZ progenitors generate clones containing both astrocytes and oligodendrocytes in vivo and mixed neuronal-glial clones in vitro (Levison and Goldman, 1993; Levison

\footnotetext{
Received June 11, 2002; revised Aug. 29, 2002; accepted Aug. 29, 2002.

This work was supported by National Institutes of Health Grant NS-17125 (J.E.G.). We thank Gord Fishell and Stewart Anderson for offering insightful comments regarding this work. We also thank Gord Fishell and Joshua Corbin for the generous gift of Dlx2/tauLacZ and Pax6 (Sey) mutant tissue, John Rubenstein and Stewart Anderson for the generous gift of $D l x 1 / 2$ and $N k x 2.1$ mutant tissue, Carol Mason for graciously sharing imaging equipment, and Peter Canoll and Ana Milosevic for critically reading this manuscript. Dritan Agalliu and Satoshi Suzuki extended advice regarding slice cultures, and Theresa Swayne offered expert assistance with confocal imaging.

Correspondence should be addressed to James E. Goldman, 630 West 168th Street, P\&S Building, \#15-420, New York, NY 10032. E-mail: jeg5@columbia.edu. Copyright (C) 2002 Society for Neuroscience $0270-6474 / 02 / 229821-10 \$ 15.00 / 0$
}

wedge of Zebrin II+ cells to form the central region of the SVZ. We used a DIx2/tauLacZ knock-in to perform a short-term lineage analysis of D/x2-expressing cells throughout SVZ formation and the postnatal peak of gliogenesis. DIx2/tauLacZ [ $\beta$-galactosidase $(\beta$-gal)]-expressing cells populate the central SVZ, whereas Zebrin II-expressing cells form its borders. Furthermore, $\beta$-gal expression demonstrates a lineage relationship between D/x2-expressing cells and glia residing in the dorsal telencephalon. We propose a model for the formation of the postnatal SVZ and demonstrate that subpallium-derived D/x2expressing cells give rise to astrocytes and oligodendrocytes in the white matter and cerebral cortex.

Key words: mouse; glia; subventricular zone; Zebrin II; DIx2; ganglionic eminence; lateral ganglionic eminence; medial ganglionic eminence and Goldman, 1997; Parnavelas, 1999). How can one reconcile the ventral specification of oligodendrocytes with the emergence of both astrocytes and oligodendrocytes from the postnatal SVZ? In one possible model, progenitors originate in the ventral telencephalon and migrate dorsally into the SVZ. Some are specified as oligodendrocyte precursors, whereas others represent astrocyte or neuronal precursors or perhaps remain uncommitted. A ventrodorsal migration of oligodendrocyte or astrocyte precursors into the dorsal telencephalon has not been demonstrated previously. Our hypothesis requires that telencephalic astrocytes and oligodendrocytes be traced back to progenitors in the embryonic ventral telencephalon.

Recent studies demonstrate tangential cell migration from the ventral basal ganglia into the dorsal cortex and hippocampus (Marin and Rubenstein, 2001). Some of these migratory progenitors express the ventral forebrain markers $D l x 1 / 2$ (Anderson et al., 1997) and give rise to interneurons, but the fate of the entire dorsally migrating population is not known. Progenitors from the ganglionic eminences have the potential to give rise to neurons and glia in vitro (He et al., 2001), but their ability to contribute astrocytes and oligodendrocytes to the dorsal telencephalon has not been demonstrated directly.

To begin examining SVZ origins, we divided the postnatal SVZ into two subpopulations of cells on the basis of the expression of Zebrin II (Aldolase C). Large, polygonal cells situated at the borders of the SVZ express Zebrin II, whereas smaller, round migratory progenitors residing in the central SVZ generally do not (Staugaitis et al., 2001). Subsequently, we characterized the expression of Zebrin II throughout embryonic and early postnatal development, observing that it is a general marker for ventricular 
zone (VZ) cells. We used a Dlx2/tauLacZ knock-in mouse (Corbin et al., 2000) to perform a short-term lineage analysis of subpallium-derived $D l \times 2$-expressing cells, following the fates of these cells and their progeny well into postnatal development. We found that cells derived from subpallial, $D l \times 2$-expressing progenitors migrate dorsally and intermix with Zebrin II-expressing VZ cells at the corticostriatal sulcus to form the dorsolateral SVZ. Furthermore, these progenitors develop into astrocytes and oligodendrocytes of the postnatal cerebral cortex and white matter.

\section{MATERIALS AND METHODS}

Animals. C57BL/J6 mice were used in all experiments requiring wildtype animals. The morning of vaginal plug formation was considered embryonic day 0 (E0) for gestational staging purposes. Mouse colonies are maintained at the Columbia University Health Sciences Campus in accordance with National Institutes of Health and United States Department of Agriculture guidelines.

Slice cultures. Organotypic slice cultures of embryonic mouse telencephalon were prepared as follows. E16 brains were dissected, embedded in a 4\% low-melting-point agarose (Bio-Rad, Hercules, CA) solution with HBSS (Invitrogen, San Diego, CA), placed directly on ice, and cut into $300 \mu \mathrm{m}$ coronal slices with a vibratome. Slices at the level of the septal nuclei were collected into ice-cold HBSS and transferred gradually into a culture medium containing BME; penicillin-streptomycin (20 $\mathrm{U} / \mathrm{ml}$; Invitrogen); L-glutamine (2 mM; Invitrogen); $5 \mu \mathrm{g} / \mathrm{ml}$ insulin, 5 $\mu \mathrm{g} / \mathrm{ml}$ transferrin, and $5 \mathrm{ng} / \mathrm{ml}$ sodium selenite (Sigma, St. Louis, MO); bovine serum albumin (1 gm fraction V; Sigma); and glucose $(0.5 \%$, Sigma). Slices were then placed onto Millicell CM tissue culture inserts (30 mm diameter, $0.4 \mu \mathrm{m}$ pore; Millipore, Bedford, MA) in six-well plates (Falcon, Franklin Lakes, NJ) containing $1.2 \mathrm{ml}$ medium and cultured in a sterile incubator $\left(5 \% \mathrm{CO}_{2}, 37^{\circ} \mathrm{C}\right)$. During the first hour after plating, slices were cultured in the defined medium listed above with $10 \%$ fetal calf serum (Invitrogen). After $1 \mathrm{hr}$, the medium was exchanged with the defined serum-free culture medium listed above with N2 and B27 supplements (1X; Invitrogen).

Retroviral infections. Retrovirus encoding cDNA for green fluorescent protein (GFP) was produced by a packaging cell line (generous gift from Drs. Suhr and Palmer, Laboratory of Genetics, The Salk Institute for Biological Studies, La Jolla, CA) that had been transfected with a retrovirus plasmid (pNIT) containing a cDNA fragment of GFP (enhanced GFP c2) (Clontech, Palo Alto, CA) and harvested as described previously (Kakita and Goldman, 1999). Virus titer was $\sim 5 \times 10^{5}$ $\mathrm{cfu} / \mathrm{ml}$. Retrovirus was drawn by capillary action into a pulled glass micropipette (Drummond, Broomall, PA), and a minimal volume $(<1 \mu \mathrm{l})$ was directed into the merged lateral ganglionic eminence (LGE) and medial ganglionic eminence (MGE) of E16 organotypic slices by hand using a dissecting microscope. Images of slices $(10 \times)$ were captured after $24 \mathrm{hr}$ in culture to detect GFP-expressing, retrovirally infected cells within the LGE/MGE using an Axioplan epifluorescence microscope with an Axiocam digital camera (Carl Zeiss, Thornwood, NY) and Openlab 3.0 imaging software (Improvision, Ltd, Lexington, MA).

Immunohistochemistry. Pregnant dams were anesthetized with the inhalent Halothane, United States Pharmacopeia (Halocarbon Labs, River Edge, NJ), and embryos were removed through a laparoscopic incision. Embryos younger than E16 were decapitated, and heads were immersion-fixed in $4 \%$ paraformaldehyde in PBS overnight at $4^{\circ} \mathrm{C}$. Embryos (E16 and older) and neonates were anesthetized with a mixture of xylazine $(75 \mathrm{mg} / \mathrm{kg}$ ) and ketamine $(5 \mathrm{mg} / \mathrm{kg}$ ) (Henry Schein, Melville, NY) and perfused transcardially with $4 \%$ paraformaldehyde in PBS. Brains were postfixed for $4 \mathrm{hr}$ at $4^{\circ} \mathrm{C}$. All tissue was cryoprotected in $30 \%$ sucrose in PBS at $4^{\circ} \mathrm{C}$, frozen at $-80^{\circ} \mathrm{C}$, cut into $16-20 \mu \mathrm{m}$ coronal sections with a cryostat, and mounted on Superfrost Plus slides (Fisher, Pittsburgh, PA). Frozen brain tissue from Dlx2/tauLacZ mice (received from Drs. J. Corbin and G. Fishell, New York University Medical Center, New York, NY) was sectioned and mounted similarly. Organotypic slices were fixed in $4 \%$ paraformaldehyde in PBS for $2 \mathrm{hr}$ at room temperature, rinsed in PBS, cryoprotected, and resliced into $25 \mu \mathrm{m}$ coronal sections for immunohistochemistry.

Immunohistochemistry with monoclonal antibodies against Zebrin II (1:100; gift from R. Hawkes, University of Calgary, Alberta, Canada), the polysialylated form of neural cell adhesion molecule (PSA-NCAM)/ 5A5 (1:5; gift from J. Dodd, Columbia University, New York, NY), RC2 (1:1; Developmental Studies Hybridoma Bank, Iowa City, IA), TUJ-1
(1:500; Babco, Richmond, CA), CNPase (1:200; Sigma), and polyclonal antibodies against Dlx2 (1:200; gift from J. Rubenstein, University of California, San Francisco, CA), astrocyte-specific glutamate transporter (GLAST) (1:1000; Chemicon, Temecula, CA), and $\beta$-galactosidase $(\beta$ gal) (1:1000; Cortex Biochemical, San Leandro, CA) was performed as follows. Cryosections were air-dried at room temperature, rinsed with PBS, and blocked in 5\% normal goat serum, $0.2 \%$ Triton X-100 in PBS for $1 \mathrm{hr}$ before incubation in primary antibodies diluted in blocking solution overnight at $4^{\circ} \mathrm{C}$. Sections were washed the following day for $1 \mathrm{hr}$ in PBS and incubated in secondary antibodies conjugated to FITC or tetramethylrhodamine isothiocyanate (TRITC) fluorochromes (Southern Biotechnology, Birmingham, AL) for 1-2 hr at room temperature. Selected sections were also rinsed in Syto-11 (1:10,000; Molecular Probes, Eugene, OR) to reveal nuclei before coverslipping in Gelmount (Biomeda, Foster City, CA). An antigen retrieval step was included for sections stained with the monoclonal antibodies to Zebrin II and CNPase: sections were incubated in $1 \mathrm{~mm}$ EDTA for $20 \mathrm{~min}$ at $65^{\circ} \mathrm{C}$ before blocking and the addition of primary antibody. A Zeiss laser scanning microscope (LSM) 510 confocal microscope and Zeiss LSM 510 imaging software were used to capture fluorescent immunoreactivity and GFP expression data from cryosections and organotypic slices.

\section{RESULTS}

\section{Zebrin II expression in the embryonic forebrain}

The Zebrin II antibody recognizes Aldolase C, a CNS-specific isoform of fructose-1,6-diphosphate aldolase expressed in astrocytes, pia mater, Bergmann glia, and Purkinje cells of adult mammals, including humans (Thompson et al., 1982; Kumanishi et al., 1985; Ahn et al., 1994; Walther et al., 1998). Zebrin II expression patterns in the developing telencephalon had not been investigated thoroughly until recently, when a partial sequence for Zebrin II was identified during a screen for genes highly expressed in the perinatal SVZ (Staugaitis et al., 2001). Zebrin II expression was detected in the border regions of the dorsolateral SVZ at postnatal day 5 (P5)-P7.

To begin characterizing the source of Zebrin II/Aldolase $C$-expressing SVZ border cells, we examined the patterns of embryonic Zebrin II expression. Zebrin II is expressed by all cells of the pseudostratified telencephalic primordium (Fig. $1 A, B)$. As neuroblasts migrate from the VZ during midgestation (Angevine and Sidman, 1961; Berry and Rogers, 1965; Rakic, 1974), they begin to express $\beta$-3-tubulin (TUJ-1) (Fig. 1C,D), a tubulin isoform specific to early differentiating neurons (Lee et al., 1990). The expression of neuronal markers is coincident with a downregulation of Zebrin II expression in the intermediate and marginal zones, as demonstrated by a temporal loss of immunoreactivity with the Zebrin II monoclonal antibody (Fig. 1E,F).

Progenitors originating in the embryonic VZ that are positive for the radial glial marker RC2 (Misson et al., 1988; Edwards et al., 1990) and the glutamate transporter GLAST (Rothstein et al., 1994; Furuta et al., 1997; Shibata et al., 1997) and possess long radial glia-like processes are immunoreactive for Zebrin II regardless of nuclear position within the developing cerebrum (Fig. $2 A, B)$. Brightly labeled Zebrin II + radial glial end feet arborize at the pial surface of the developing cortex (Fig. 2C,D). Furthermore, process-bearing cells in the developing cortex and striatum coexpress Zebrin II along with RC2, probably representing radial glia transforming into mature, stellate astrocytes (Misson et al., 1988; Voigt, 1989) (Fig. 2E,F). Zebrin II/Aldolase C expression is specific to mature astrocytes in the forebrain (Thompson et al., 1982; Kumanishi et al., 1985; Ahn et al., 1994; Walther et al., 1998; Staugaitis et al., 2001). Hence, we infer that astrocytes originating directly from the neuroepithelium and indirectly via a radial-glial phenotype maintain the expression of Zebrin II during differentiation. 

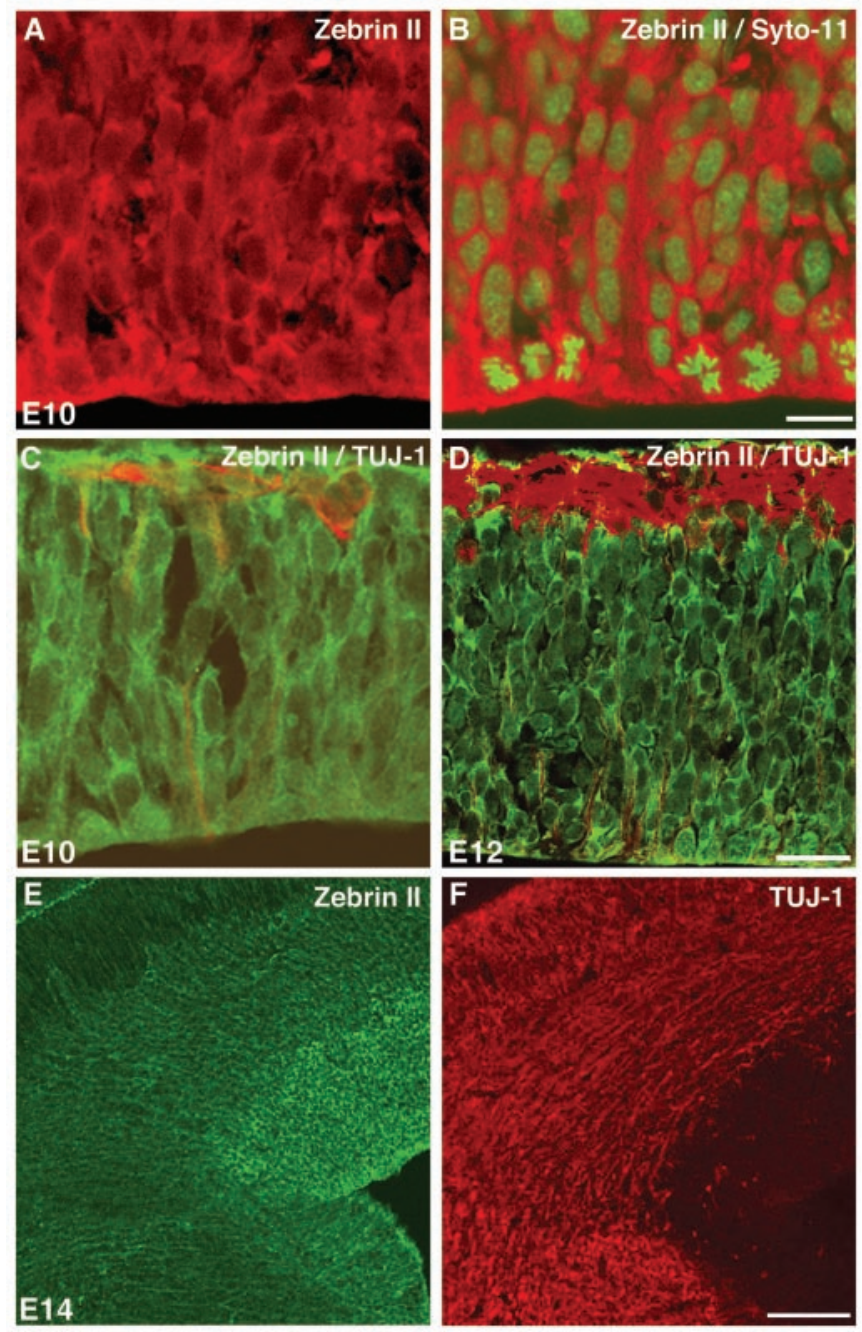

Figure 1. Zebrin II is expressed by cells of the telencephalic primordium. $A, B$, Coronal sections $(20 \mu \mathrm{m})$ of E10 cortical ventricular zone at the level of the septal nuclei show Zebrin II immunoreactivity (TRITC); Syto-11 (FITC) reveals nuclei and mitotic activity of Zebrin II + cells at the surface of the lateral ventricle. $C, D$, Neuronal precursors coexpress Zebrin II (FITC) and TUJ-1 (TRITC) within the neuroepithelium at E10 and E12. $E, F$, TUJ-1+ neurons lose Zebrin II immunoreactivity after translocating or migrating from the ventricular zone at E14. Scale bars: $B$, $10 \mu \mathrm{m} ; D, 20 \mu \mathrm{m} ; F, 200 \mu \mathrm{m}$.

\section{Zebrin II-expressing VZ cells intermix with DIx2- expressing cells at the corticostriatal boundary}

As the LGE enlarges during midgestation (E12-E14), the pallial VZ "zippers up" (folds at an acute angle) during corticostriatal sulcus formation, generating a wedge-shaped zone of Zebrin II+ VZ cells (Fig. 3A,D). During the subsequent perinatal week, this area undergoes a transformation such that Zebrin II-expressing cells become distributed at the medial, dorsal, and lateral periphery of the SVZ, with a large number of Zebrin II-negative cells populating the central region (Staugaitis et al., 2001). We hypothesized that these Zebrin II-negative cells originate in more ventral locations and migrate dorsally into the SVZ.

As one test of our hypothesis, we studied the position of Dlx2-expressing progenitors relative to the Zebrin II + VZ cells from midembryogenesis through postnatal stages. Dlx2immunoreactive cells collected at the ventral aspect of the Zebrin II wedge by E14-E16 but did not themselves express Zebrin II
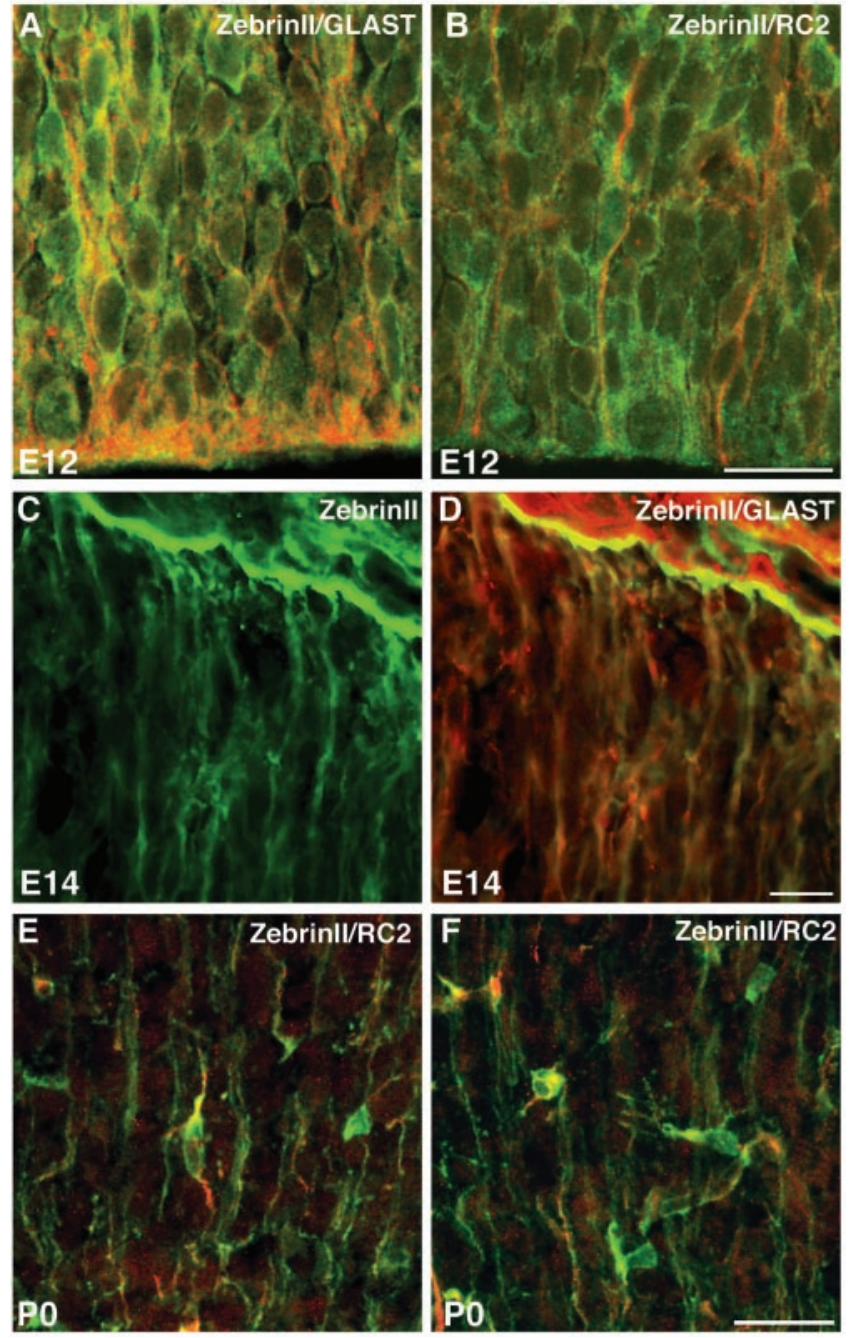

Figure 2. Zebrin II is expressed by glial progenitors regardless of nuclear position. $A, B$, Zebrin II + cells (FITC) coexpress GLAST and RC2 (both TRITC) within the neuroepithelium at E12. $C, D$, Radial glial end feet coexpress Zebrin II (FITC) and GLAST (TRITC) at E14. E, F, Processbearing cells within the developing cerebral cortex coexpress Zebrin II (FITC) and RC2 (TRITC) and demonstrate the morphology of differentiating astrocytes at P0. Scale bars: $B, 20 \mu \mathrm{m} ; D, 10 \mu \mathrm{m} ; F, 40 \mu \mathrm{m}$.

(Fig. 3B). A relative minority of Dlx2-expressing cells appeared to be scattered within and just dorsal to the Zebrin II+ wedge by this time; however, a large majority of cells remained ventral to the wedge at E16. By E19/P0, D1x2 expression was still found in the LGE but was also in central portions of the dorsolateral SVZ and in the overlying white matter (Fig. $3 C$ ). Hence, Dlx2+ cells populated at least a portion of the forming dorsolateral SVZ during perinatal development, corresponding precisely to the late, medial migration pathway described previously (Anderson et al., 2001).

\section{Cells derived from Dlx2-expressing progenitors are positive for PSA-NCAM}

Dlx2-expressing cells did not account for all of the Zebrin IInegative cells within the dorsolateral SVZ. Hence, we studied the expression pattern of molecular markers present within the developing SVZ and examined the PSA-NCAM, for two reasons. First, PSA-NCAM is expressed by the majority of perinatal SVZ cells that ultimately differentiate into astrocytes and oligodendro- 
Figure 3. The VZ zippers up at the corticostriatal boundary by E14. $A$, Fasciculating fibers emanate from Zebrin II+ (FITC) cells at the E14 lateral VZ. $B$, Dlx2 immunoreactive cells (FITC) are dispersed throughout the LGE and collect at the Zebrin II+ (TRITC) wedge of VZ at E16. $C$, By E19/P0, Dlx2+ cells (FITC) reside in the LGE, dorsolateral SVZ, and overlying white matter. $D$, The VZ zippers up, by folding at an acute angle during corticostriatal sulcus formation. Scale bars: $A, B, 100$ $\mu \mathrm{m} ; C, 50 \mu \mathrm{m}$.
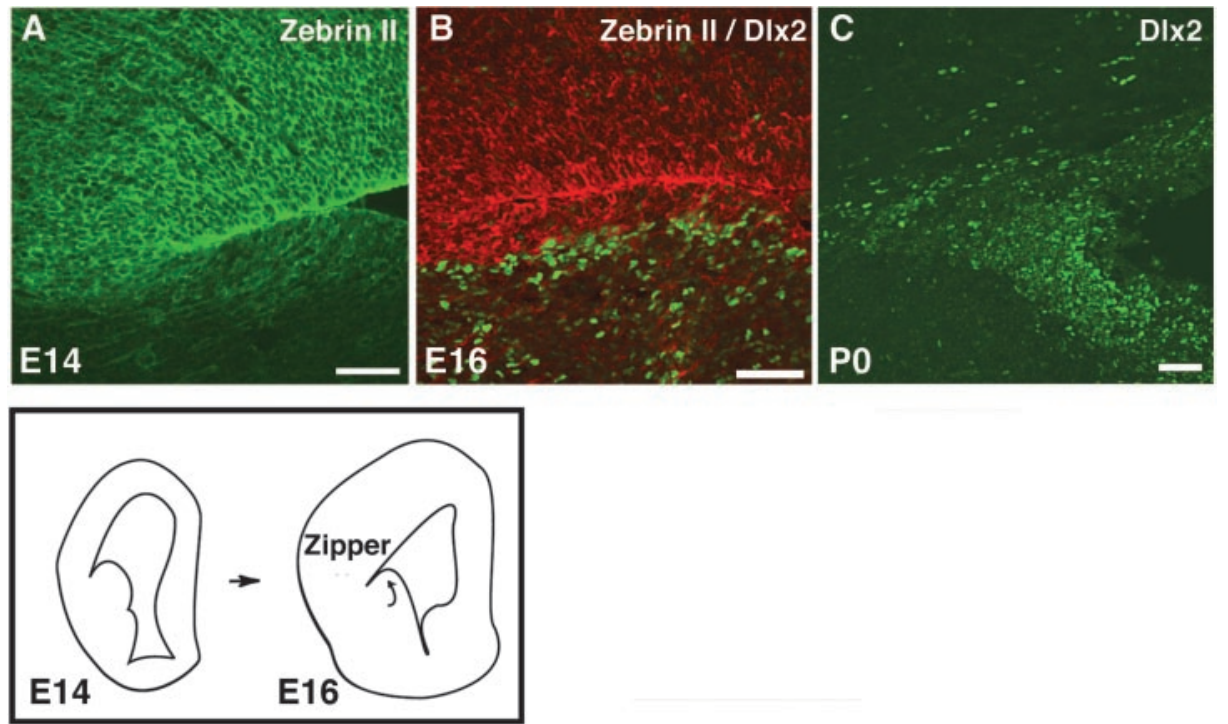

cytes in vivo (Levison et al., 1993) and on transplantation from the adult SVZ into demyelinating lesion sites within the CNS (Keirstead et al., 1999; Nait-Oumesmar et al., 1999). Second, PSA-NCAM expression has also been associated with migratory SVZ neuroblasts (Doetsch and Alvarez-Buylla, 1996; Bruses and Rutishauser, 2001). We found that many PSA-NCAM-expressing cells also collected at the ventral limit of the Zebrin II + wedge by E14-E16 and, like the Dlx2+ cells that collected here, did not themselves express Zebrin II (Fig. $4 A-C$ ). Many PSA-NCAM+ cells extended leading processes directed dorsally toward the cortex and possessed the simple unipolar or bipolar morphology common to migratory progenitors in this region studied previously with time-lapse microscopy (Fig. 4B,C) (Kakita and Goldman, 1999). By P0, the central Zebrin II-negative region of the SVZ was populated primarily by PSA-NCAM-positive cells (see below).
Figure 4. PSA-NCAM + cells are the progeny of Dlx2-expressing cells. By E16, PSANCAM-expressing cells also collect at the ventral aspect of the Zebrin II+ VZ wedge, the site of the nascent dorsolateral SVZ. $A-C$, Immunoreactivity for PSA-NCAM (TRITC) and Zebrin II (FITC) at the acute dorsolateral angle of the lateral ventricle. $B$, $C$, asterisk, The PSA-NCAM+ cell extends leading processes ventrodorsally. Dlx2/tauL$a c Z$ ( $\beta$-gal) labels PSA-NCAM + progeny of Dlx2-expressing cells at the ventral aspect of the VZ. $D-H$, Immunoreactivity for $\beta$-galactosidase (TRITC) and PSA-NCAM (FITC) within the LGE at E16 $(D-F)$ and forming dorsolateral SVZ at P0 $(G-H) . H$, Enlargement of boxed area in $G$. Scale bars: $B, 50 \mu \mathrm{m} ; E, G, 100 \mu \mathrm{m}$.
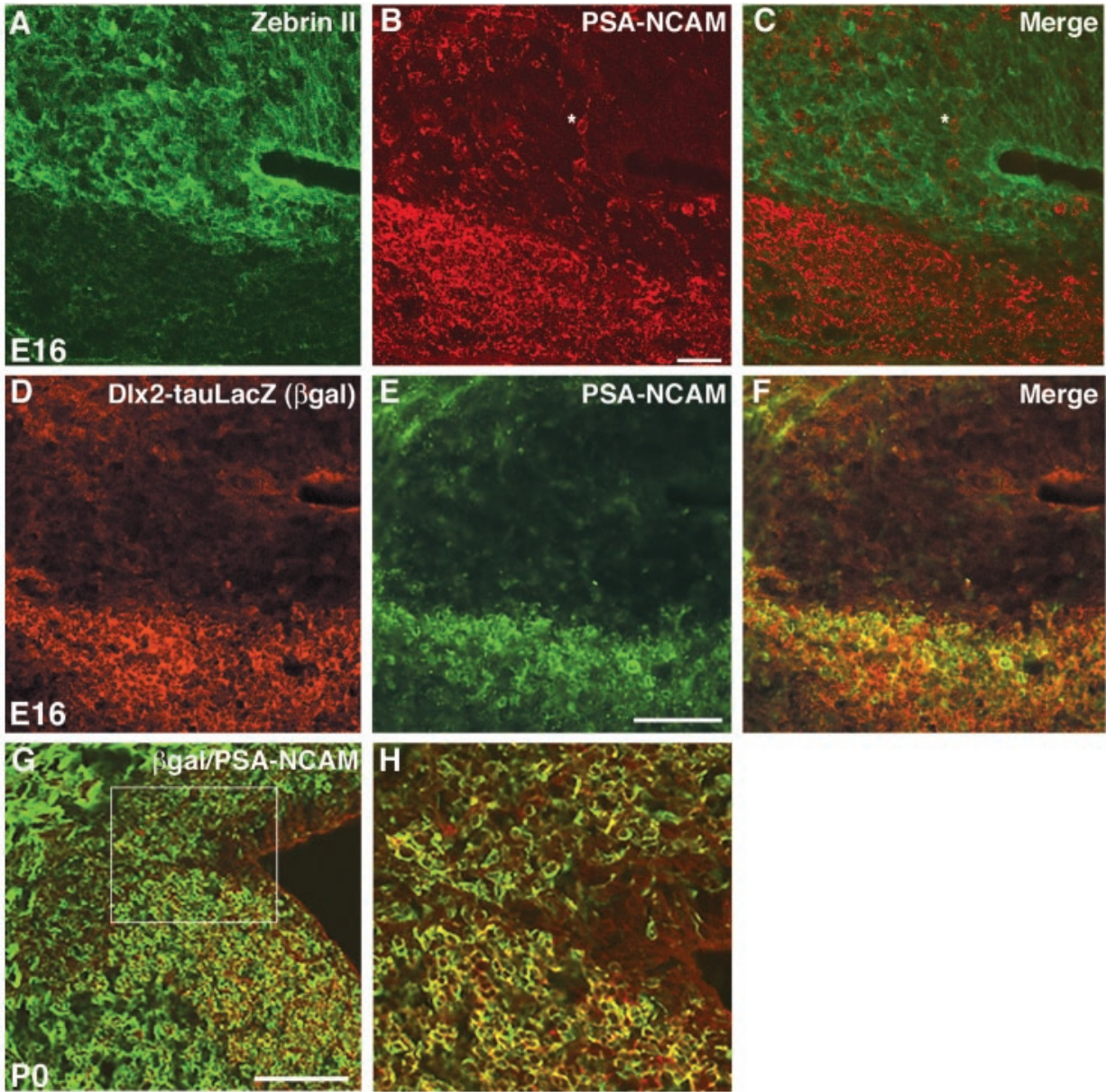

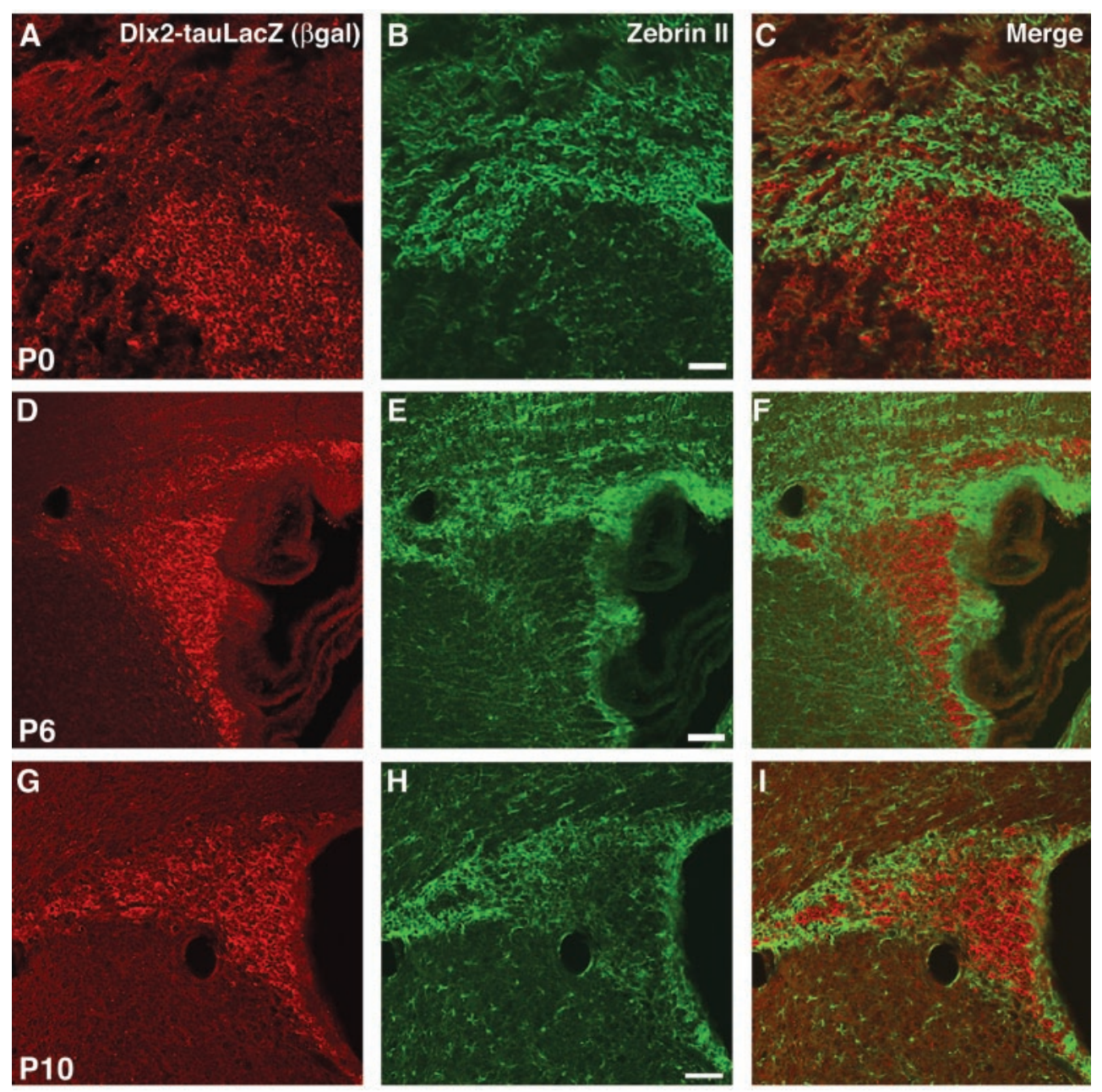

Figure 5. Dlx2-expressing cells and their progeny, defined by $D l x 2 /$ tauLacZ ( $\beta$-gal) expression, intermix with Zebrin II+ VZ cells to form the dorsolateral SVZ. $A-C$, $\beta$-gal (TRITC)- and Zebrin II (FITC)immunoreactive cells begin to mix perinatally. $D-I$, Postnatally, Zebrin II + cells are displaced to form the outer limits of the SVZ, whereas $\beta$-gal+ cells constitute the Zebrin II- central region at P6 $(D-F)$ and P10 $(G-I)$. Note that in $A$ and $D, \beta$-gal + cells also stream into the overlying white matter. Scale bars: $B, 100$ $\mu \mathrm{m} ; E, 50 \mu \mathrm{m} ; H, 20 \mu \mathrm{m}$.
None of the PSA-NCAM-positive cells within the LGE and perinatal SVZ also expressed Dlx2. Hence, it was unclear whether these two distinct populations shared common origins. Using a Dlx2/tauLacZ knock-in mouse (Corbin et al., 2000), we performed a short-term in vivo lineage analysis of the Dlx2expressing cell population within the perinatal LGE and SVZ. Using antibodies to $\beta$-gal and PSA-NCAM, we found a large overlap in expression of the two markers within the LGE at E16 (Fig. 4D-F) and presumptive dorsolateral SVZ at P0 (Fig. $4 G, H)$. Thus, cells that downregulated the Dlx2 protein but retained the relatively stable tauLacZ ( $\beta$-gal) reporter also expressed PSA-NCAM, demonstrating a direct lineage relationship between the two cell populations.

\section{Cells defined by Zebrin II or DIx2/tauLacZ expression form the postnatal SVZ}

Extensive cell proliferation and mixing during the perinatal week results in a heterogeneous assortment of postnatal SVZ cells; however, the complex mixture could be delineated by Zebrin II and $\beta$-gal expression. The VZ wedge becomes fenestrated as cells from ventral areas invade it, with the most dorsolateral Zebrin II + cells becoming "displaced" from more medial VZ wedge cells. Analysis of the Zebrin II expression pattern at each day throughout the perinatal and early postnatal weeks reveals that the accumulation of these migrating cells progressively displaces the Zebrin II-positive residual neuroepithelium laterally (data not shown). This embryological displacement should not be con- fused with migration. We find no evidence suggesting that these wedge cells are migratory. Rather, the lateral wedge cells, which form the dorsolateral tip of the SVZ, appear to be relatively stationary. Stationary Zebrin II + SVZ border cells are characterized by "large polygonal cell bodies with several fine processes," resembling the light border cells described previously by Smart (1961) (Staugaitis et al., 2001). These cells are morphologically and molecularly distinct from those displaying small, round cell bodies with dark nuclei and one or two fine processes that populate the central SVZ (Smart, 1961). The central SVZ population was shown to be relatively more proliferative and migratory than border cells and to be generally devoid of Zebrin II mRNA and protein expression (Staugaitis et al., 2001).

The collection of Dlx2-expressing cells with their descendants, as defined by $D l \times 2 /$ tauLacZ expression, constituted the vast majority of Zebrin II-negative cells in the forming perinatal SVZ (Fig. $5 A-C$ ). By P6-P10, a $\beta$-gal+ subpopulation was positioned centrally within the dorsolateral SVZ, whereas Zebrin II+ residual VZ cells formed its outer boundaries (Fig. $5 D-I$ ). $\beta$-gal+ cells appear in the overlying white matter and cortex (Fig. 5D,F) (see below).

\section{PSA-NCAM-expressing progenitors migrate ventrodorsally from the ganglionic eminences into the dorsolateral SVZ}

The migratory behavior of Dlx2-expressing cells has been demonstrated previously (Anderson et al., 1997, 2001). The pattern of 

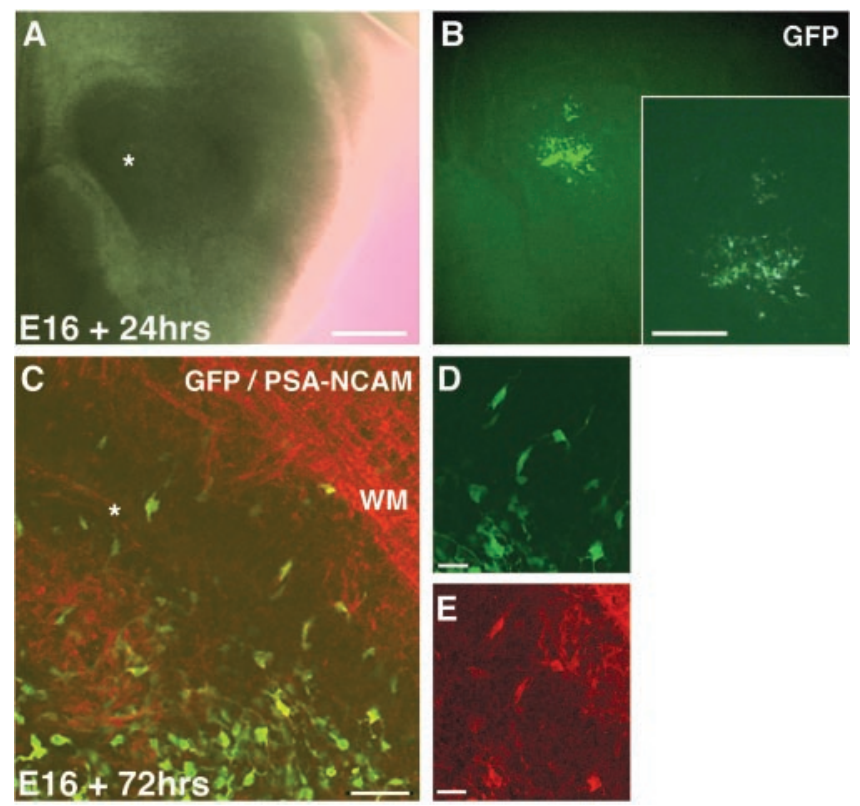

Figure 6. Migration assays in organotypic slice cultures confirm ventrodorsal migration of PSA-NCAM-expressing cells into the SVZ. A, Retrovirus was injected into the merged LGE/MGE of organotypic slices harvested from E16 telencephalon at the level of the septal nuclei. Asterisk, Site of injection. $B$, GFP expression by infected LGE/MGE cells after $24 \mathrm{hr}$ in culture. $B$, inset, Cells migrate radially into the developing striatum and tangentially or ventrodorsally toward the VZ wedge. $C-E$, GFP-expressing cells that migrate into the dorsolateral SVZ show immunoreactivity for PSA-NCAM (TRITC) after $72 \mathrm{hr}$ in culture. C, asterisk, Dorsolateral tip of the lateral ventricle. $W M$, White matter. Scale bars: $A$, $500 \mu \mathrm{m} ; B$ (inset), $300 \mu \mathrm{m} ; C-E, 50 \mu \mathrm{m}$.

PSA-NCAM expression within the ganglionic eminences and perinatal SVZ suggested that the PSA-NCAM+ population also invades the VZ wedge during the formation of the perinatal SVZ. However, an assay of cell migration was necessary to demonstrate the dynamic invasion as opposed to a gradual expansion or ventrodorsal wave of PSA-NCAM expression by cells within the developing SVZ. A retrovirus encoding the cDNA for GFP was injected into the lateral and medial ganglionic eminences (merged LGE/MGE) of organotypic slices harvested from embryos at E16. At E16, the LGE and MGE at the level of the septal nuclei are fused and cannot be targeted individually by retroviral labeling strategies. Hence, we predict that our migration assays included labeled cells from both the MGE and LGE. After $24 \mathrm{hr}$, slices were imaged to detect the location of GFP-expressing cells within the merged LGE/MGE structure $(n=16)$ (Fig. 6A,B). Slices were cultured up to $72 \mathrm{hr}$ to allow migration to occur before being fixed, cryoprotected, and resliced into thin sections for immunolabeling with a monoclonal antibody to PSA-NCAM $(n=$ 14). PSA-NCAM+, GFP-expressing cells were identified within the perinatal SVZ of each slice (Fig. $6 C-E$ ), demonstrating the dorsal migratory nature of this population of cells and their ability to invade the VZ wedge during the perinatal period. Therefore, two populations of cells, identified either by Dlx 2 or PSA-NCAM protein expression, invade the Zebrin II+ VZ wedge to form collectively the central dorsolateral SVZ.

\section{DIx2/tauLacZ defined cells give rise to astrocytes and oligodendrocytes in the dorsal telencephalon}

To determine whether any of the $\beta$-gal + cells migrated into the dorsal telencephalon to become glia, we looked for coexpression of $\beta$-gal and glial markers. Indeed, $\beta$-gal + cells within the striatum, white matter, and cortex developed into astrocytes and oligodendrocytes (Fig. 7). Some cells in white matter that possessed an immature phenotype characteristic of migratory SVZ progenitors (Kakita and Goldman, 1999) coexpressed $\beta$-gal and Zebrin II (Fig. $7 A-C$ ). We infer that these cells are early astrocytes, as Zebrin II-negative cells migrate from the central SVZ and initiate Zebrin II expression along with vimentin and glial fibrillary acidic protein (GFAP) as they differentiate into astrocytes (Staugaitis et al., 2001). Other $\beta$-gal+ cells exhibited a more differentiated astrocytic phenotype, coexpressing GFAP and associating with blood vessels (Fig. $7 D-F$ ). In addition, other $\beta$-gal + cells developed the morphological and molecular phenotype of mature oligodendrocytes in the white matter (Fig. 7G-I) and cortex (Fig. $7 J-L)$. They possessed large somas, long processes of uniform width that were oriented along fiber tracts, and coexpressed the oligodendrocyte-specific enzyme CNPase.

\section{DISCUSSION}

In this study, we identified the embryonic origins of two distinct populations of SVZ cells. Zebrin II+ residual ventricular zone cells form the borders of the SVZ. Cells sharing a Dlx2expressing, subpallial origin migrate along a medial ventrodorsal pathway to populate the central SVZ and give rise to astrocytes and oligodendrocytes in the developing cerebral cortex and white matter (Fig. 8, model).

\section{Zebrin II: a novel marker for cells of the ventricular zone}

Zebrin II is expressed uniformly throughout the dorsoventral axis of the telencephalon until E10, when differentiation of the basal forebrain and cerebral cortex begins (Fig. 8, E10) (Angevine and Sidman, 1961; Berry and Rogers, 1965; Rakic, 1974; Eisenstat et al., 1999; Wilson and Rubenstein, 2000). Zebrin II expression appears to be a quality common to all telencephalic VZ cells, including neuroblasts and glioblasts, and does not appear to be regulated by the dorsoventral patterning genes $N k x 2.1, D l \times 1 / 2$, or Pax6. For example, the pattern of Zebrin II expression is not disrupted in $N k x 2.1$ or $D l x 1 / 2$ mutants (our unpublished observations); hence, it does not appear to be regulated by these transcription factors. In small-eye (Sey) mice in which the Pax6 gene is disrupted, radial glia are not generated; hence, the overall number of dorsal VZ cells is diminished (Hill et al., 1991; Stoykova et al., 1996). However, Zebrin II is still expressed by remaining VZ cells (our unpublished observations).

\section{Origins of astrocytes}

Two temporospatially distinct sources of astrocytes exist within the developing forebrain. Embryonically, astrocytes are generated from the ventricular zone (Luskin et al., 1988; Price and Thurlow, 1988) via a radial glial intermediate phenotype (Voigt, 1989). We argue that these astrocytes retain Zebrin II expression throughout their differentiation. The postnatal SVZ serves as another source of forebrain astrocytes (Smart, 1961; Lewis, 1968; Privat and Leblond, 1972; Paterson et al., 1973; Levison and Goldman, 1993; Luskin and McDermott, 1994), giving rise to a second class of astrocyte precursor cell. These precursors emerge from Zebrin II-negative cells within the postnatal SVZ, initiate Zebrin II expression, and differentiate into mature astrocytes within the striatum, white matter, and cortex (Staugaitis et al., 2001). Although astrocytes derived from both sources express GFAP and associate with blood vessels, it is possible that they possess qualities making them distinct from one another. The 

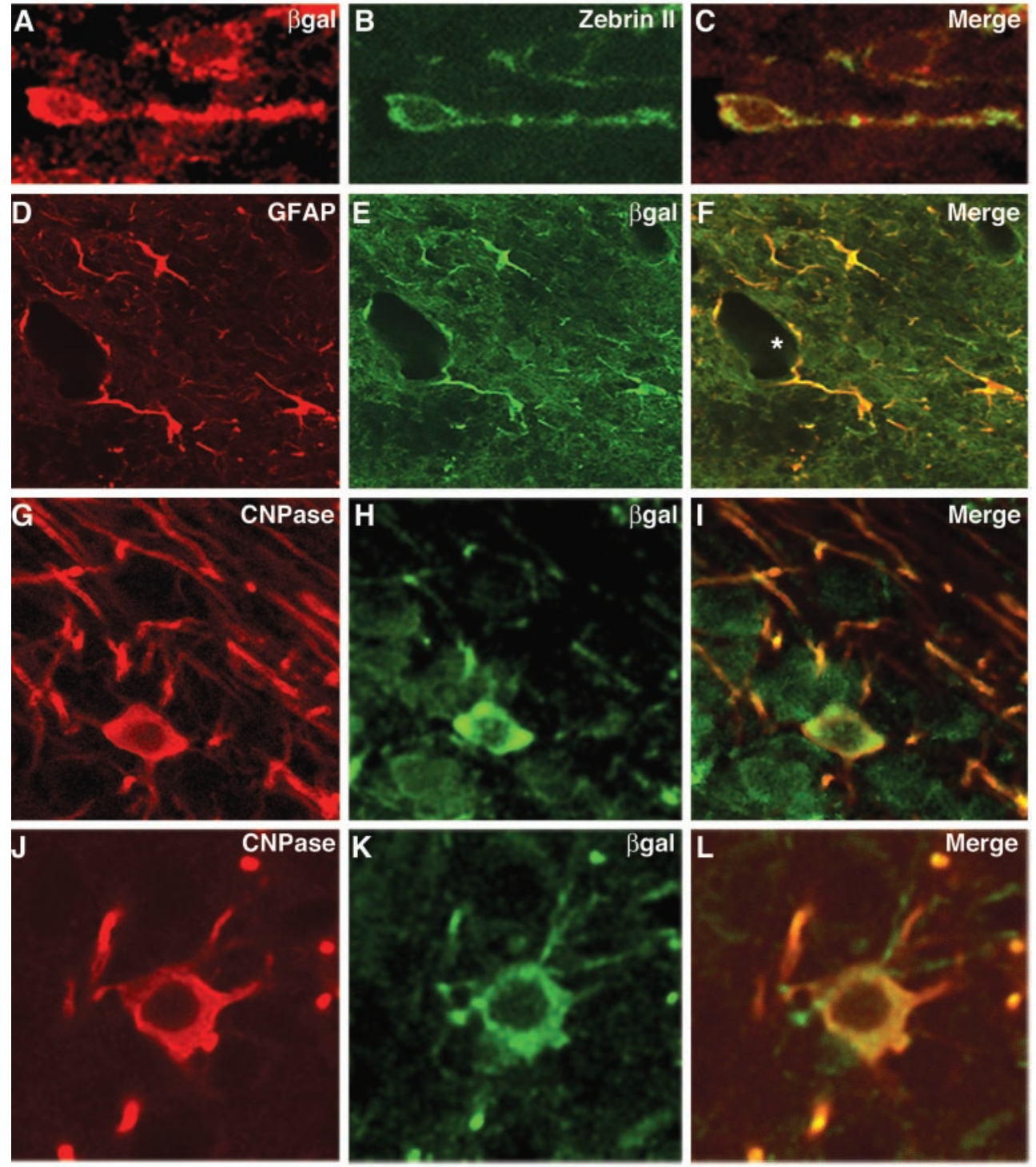

Figure 7. Subpallium-derived cells defined by Dlx2/tauLacZ reporter expression adopt mature glial phenotypes in the postnatal dorsal forebrain (P6-P10). $A-C$, Immature $\beta$-gal + cells (TRITC) positioned within the corpus callosum coexpress Zebrin II (FITC). $D-F, \beta$-gal+ cells (FITC) exhibiting a more differentiated astrocytic phenotype coexpress GFAP (TRITC) and associate with blood vessels (note asterisk in $F$ ). $\beta$-gal+ cells coexpress CNPase and possess the morphology of mature oligodendroglia in the white matter $(G-I)$ and cerebral cortex $(J-L)$. comparison of diverse classes among Zebrin II-expressing astrocytes may enable us to understand better the roles astrocytes serve in the normal and diseased CNS.

\section{Origins of oligodendrocytes}

Recent work has described a ventral origin and specification of telencephalic oligodendrocytes during embryonic development (Woodruff et al., 2001). Before birth, progenitors isolated from the rat striatum have a much greater competence to generate oligodendrocytes in vitro than those harvested from cerebral cortex. However, progenitors from the postnatal cerebral cortex have the potential to give rise to significant numbers of oligodendrocytes (Birling and Price, 1998). One popular interpretation of this phenomenon is that all oligodendrocyte precursors are specified by Shh and migrate into the dorsal telencephalon during the course of embryogenesis (Woodruff et al., 2001). This interpretation is based on studies in which a gradient of DM-20, an alternatively spliced isoform of myelin proteolipid protein, and platelet-derived growth factor receptor- $\alpha$ (PDGFR- $\alpha$ ) expression emanates from the boundary separating the anterior hypothalamus and MGE and extends into the dorsal telencephalon (Pringle and Richardson, 1993; Spassky et al., 1998; Tekki-Kessaris et al.,
2001). The migratory nature of these DM-20+/PDGFR- $\alpha+$ cells has been inferred from the ability of oligodendrocyte precursors in the optic nerve to migrate long distances (Ono et al., 1997) but has not been demonstrated directly. The ventrodorsal gradient of DM-20 and PDGFR- $\alpha$ expression could represent the migration of such cells or the acquisition of these markers by some precursors during the course of their migration. Alternatively, some cells might remain DM-20/PDGFR- $\alpha$-negative during migration and express these markers only once they have settled in the dorsal white matter or cortex. Hence, some ventrally originating progenitors, which are competent to form oligodendrocytes but do not yet express common oligodendrocyte-specific markers, may migrate dorsally into the forming dorsolateral SVZ. Regardless of the precise stage at which ventrally derived cells begin to express oligodendrocyte-specific markers, it is clear that gliogenesis is well under way throughout the developing cerebrum by the day of birth, with the peak of oligodendrocyte and astrocyte generation occurring during the first 2 postnatal weeks.

We observed very little PDGFR- $\alpha$ expression by cells migrating along a medial tangential pathway from the ganglionic eminence or by cells within the dorsolateral SVZ itself (data not 


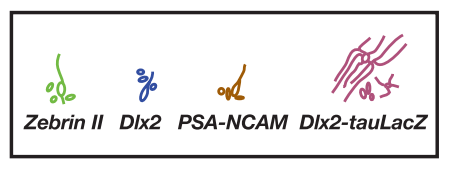

Figure 8. Formation of the postnatal SVZ. E10, Zebrin II is expressed by all cells of the telencephalic primordium. E14, A Zebrin II+ wedge emerges during corticostriatal sulcus formation. E16, E16 Box, The Zebrin II+ wedge is fenestrated by Dlx2+ or PSANCAM + migratory, subpallium-derived cells. PSA-NCAM+ cells are the progeny of Dlx $2+$ cells. At $\mathrm{P} 0$, the total population of Dlx2-expressing cells and their PSANCAM+ progeny can be defined by $D l \times 2 /$ tauLacZ ( $\beta$-gal) expression. Subpalliumderived cells defined by $D l x 2 /$ tauLacZ $(\beta$-gal) expression form the central region of the perinatal SVZ. At P10 (and for the P10 box), the postnatal SVZ can be delineated into two subpopulations of cells based on the expression of Zebrin II or the Dlx2/tauLacZ reporter. Zebrin II+ residual VZ cells form the outer borders of the SVZ. Cells sharing a Dlx2-expressing subpallial origin, as defined by $D l \times 2 /$ tauLacZ ( $\beta$-gal) expression, populate the central SVZ and give rise to astrocytes (astros) and oligodendrocytes (oligos) in the striatum, white matter, and cortex. Note: model is not drawn to scale.
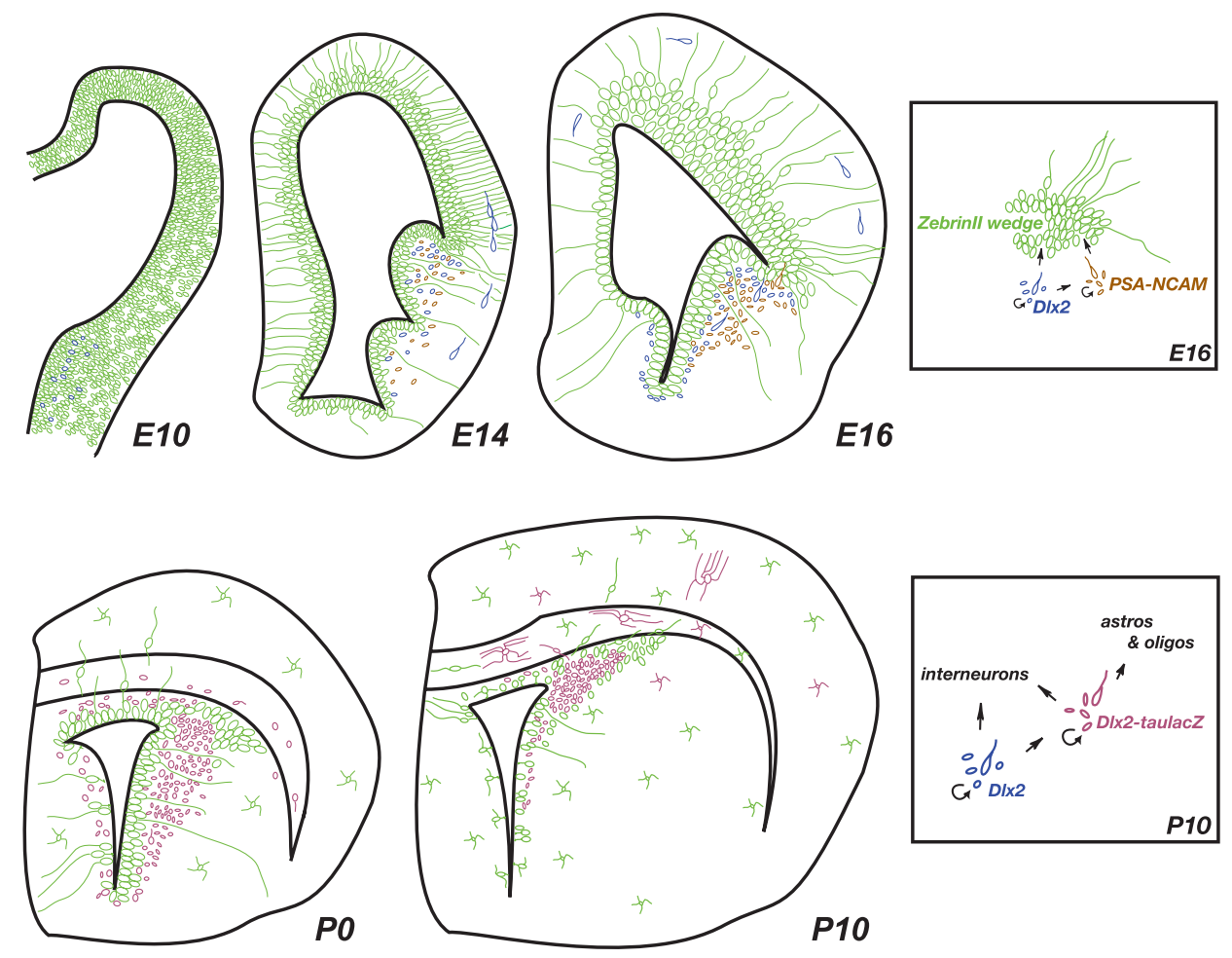

shown). However, a subset of cells that were retrovirally labeled within the SVZ and have migrated into the overlying white matter do begin to express PDGFR- $\alpha$, along with other markers thought to be specific to developing oligodendrocytes (J. Power, unpublished observations). This observation is consistent with previous work (Pringle et al., 1992) demonstrating a lack of PDGFR- $\alpha$ expression by SVZ cells and an upregulation of the receptor by cells within the surrounding parenchyma. Although PDGFR- $\alpha$ expression may identify oligodendrocyte precursors in the beginning stages of differentiation, the identification of many migratory oligodendrocyte precursors could be more elusive. It is possible that ventrally originating cells become competent to give rise to oligodendrocytes by exposure to extrinsic patterning factors such as Shh without committing to an oligodendrocyte lineage or necessarily expressing early oligodendrocyte markers such as DM-20 or PDGFR- $\alpha$. These "oligocompetent" cells may remain as multipotent progenitors or glioblasts until they have migrated dorsally and established residence within the SVZ. Alternatively, extrinsic factors within the postnatal SVZ, or even within the white matter or cortex, might serve to specify oligodendrocytes locally.

We demonstrate that a population of cells derived from Dlx2expressing cells within the ganglionic eminences migrates within the medial tangential pathway (Anderson et al., 2001) into the dorsolateral SVZ. Extensive study of the expression of patterning genes in the developing forebrain led us to believe that cells expressing $D l x$ genes originate within the subpallium (Eisenstat et al., 1999; Puelles et al., 2000). The proportion of cells from this population that differentiate into glial cells rather than interneu- rons is unclear. We did not observe any cells that coexpressed Dlx2 protein and oligodendrocyte-specific markers. We infer that oligodendrocyte differentiation begins after Dlx2 protein expression is downregulated by subpallium-derived precursors. Stable Dlx2-tauLacZ reporter expression enabled us to trace the lineage of $D l x 2$-expressing cells into later stages that overlapped with oligodendrocyte differentiation. We observed Dlx2-tauLacZ ( $\beta$ gal) expression in a minority of glial cells in the white matter and cortex. As glioblasts proliferate during migration, the $\beta$-gal reporter may become titrated in glial sublineages below levels detectable by immunohistochemistry. The reporter might also become degraded before the expression of glia-specific markers by a large number of cells. Additional lineage analyses performed in vivo are necessary to determine the lineal relationship among interneurons and glia within the Dlx2-expressing progenitor population.

Cells within the white matter of the perinatal telencephalon were found to be coimmunoreactive for a pan-Dlx antibody and an antibody to the NG2 proteoglycan expressed by oligodendrocyte precursors (He et al., 2001). These data suggest that these oligodendrocytes coexpress Dlx1/2 or Dlx5/6 and imply that they were derived from Dlx1/2-expressing LGE cells. However, a direct lineage relationship was not demonstrated in vivo. Our results show that subpallium-derived $D l \times 2$-expressing cells and their progeny, as defined by Dlx2-tauLacZ ( $\beta$-gal) expression, populate the central zone of the dorsolateral SVZ during the first postnatal week. Furthermore, we show that these cells give rise to oligodendrocytes and astrocytes in the developing cerebrum. 


\section{Sublineages within the postnatal SVZ}

The SVZ is composed of a heterogeneous mixture of cells from a variety of different lineages. Some SVZ cells become specified as astroblasts or oligodendroblasts (Levison and Goldman, 1993; Luskin and McDermott, 1994; Parnavelas, 1999). Others remain uncommitted as glioblasts until they migrate into the overlying parenchyma, where they diverge into lineages of astrocytes or oligodendrocytes (Levison et al., 1993) (M. Zerlin, unpublished observations). Many SVZ cells appear to commit to a neuronal lineage and take a rostral migratory route toward the olfactory bulb, where they give rise to interneurons (Luskin, 1993; Lois and Alvarez-Buylla, 1994). A subset of SVZ cells may remain multipotent. Clonal analyses of progenitors isolated from the LGE (He et al., 2001) and the postnatal dorsolateral SVZ (Levison and Goldman, 1997) demonstrate the potential of many of these immature cells to give rise to both neurons and glia in vitro. Hence, fate specification may occur within the SVZ or even after emigration from the SVZ. In vivo, instructive or permissive factors enable some precursor cells to migrate into the developing cerebrum, whereas others remain within the SVZ. The extent to which a relationship exists between migration pathways and fate specification remains unknown. An understanding of the mechanisms behind telencephalic neuronal-glial specification and the release of progenitors from the SVZ is essential to better understand gliogenesis within the embryonic and postnatal forebrain.

\section{REFERENCES}

Ahn AH, Dziennis S, Hawkes R, Herrup K (1994) The cloning of zebrin II reveals its identity with aldolase C. Development 120:2081-2090.

Anderson SA, Eisenstat DD, Shi L, Rubenstein JLR (1997) Interneuron migration from basal forebrain to neocortex: dependence on Dlx genes. Science 278:474-476.

Anderson SA, Marin O, Horn C, Jennings K, Rubenstein JLR (2001) Distinct cortical migrations from the medial and lateral ganglionic eminences. Development 128:353-363.

Angevine Jr JB, Sidman RL (1961) Autoradiographic study of cell migration during histogenesis of cerebral cortex in the mouse. Nature 192:766-768.

Berry M, Rogers AW (1965) The migration of neuroblasts in the developing cerebral cortex. J Anat 99:691-709.

Birling MC, Price J (1998) A study of the potential of the embryonic rat telencephalon to generate oligodendrocytes. Dev Biol 193:100-113.

Bruses JL, Rutishauser U (2001) Roles, regulation, and mechanism of polysialic acid function during neural development. Biochimie 83:635-643.

Corbin JG, Gaiano N, Machold RP, Langston A, Fishell G (2000) The GSH2 homeodomain gene controls multiple aspects of telencephalic development. Development 127:5007-5020.

Doetsch F, Alvarez-Buylla A (1996) Network of tangential pathways for neuronal migration in adult mammalian brain. Proc Natl Acad Sci USA 93:14895-14900.

Edwards MA, Yamamoto M, Caviness Jr VS (1990) Organization of radial glial and related cells in the developing murine CNS: an analysis based upon a new monoclonal antibody marker. Neuroscience 36:121-144.

Eisenstat DD, Liu JK, Mione M, Zhong W, Yu G, Anderson SA, Ghattas I, Puelles L, Rubenstein JLR (1999) Dlx-1, Dlx-2, and Dlx-5 expression define distinct stages of basal forebrain differentiation. J Comp Neurol 414:217-237.

Furuta A, Rothstein JD, Martin LJ (1997) Glutamate transporter protein subtypes are expressed differentially during rat CNS development. J Neurosci 17:8363-8375.

He W, Ingraham C, Rising L, Goderie S, Temple S (2001) Multipotent stem cells from the mouse basal forebrain contribute GABAergic neurons and oligodendrocytes to the cerebral cortex during embryogenesis. J Neurosci 21:8854-8862.

Hill RE, Favor J, Hogan BL, Ton CC, Saunders GF, Hanson IM, Prosser J, Jordan T, Hastie ND, van Heyningen V (1991) Mouse small eye results from mutations in a paired-like homeobox-containing gene. Nature 354:522-525.

Kakita A, Goldman JE (1999) Patterns and dynamics of SVZ cell migration in the postnatal forebrain: monitoring living progenitors in slice preparations. Neuron 23:461-472.

Keirstead HS, Ben-Hur T, Rogister B, O'Leary MT, Dubois-Dalcq M,
Blakemore WF (1999) Polysialylated neural cell adhesion moleculepositive CNS precursors generate both oligodendrocytes and Schwann cells to remyelinate the CNS after transplantation. J Neurosci 19:7529-7536.

Kumanishi T, Watabe K, Washiyama K (1985) An immunohistochemical study of aldolase $\mathrm{C}$ in normal and neoplastic nervous tissues. Acta Neuropathol (Berl) 67:309-314.

Lee MK, Tuttle JB, Rebhun LI, Cleveland DW, Frankfurter A (1990) The expression and posttranslational modification of a neuron-specific beta-tubulin isotype during chick embryogenesis. Cell Motil Cytoskel $17: 118-132$.

Levison SW, Goldman JE (1993) Both oligodendrocytes and astrocytes develop from progenitors in the subventricular zone of postnatal rat forebrain. Neuron 10:201-212.

Levison SW, Goldman JE (1997) Multipotential and lineage restricted precursors coexist in the mammalian perinatal subventricular zone. J Neurosci Res 48:83-94.

Levison SW, Chuang C, Abramson BJ, Goldman JE (1993) The migrational patterns and developmental fates of glial precursors in the rat subventricular zone are temporally regulated. Development 119:611-622.

Lewis PD (1968) The fate of the subependymal cell in the adult rat brain, with a note on the origin of microglia. Brain 91:721-738.

Lois C, Alvarez-Buylla A (1994) Long-distance neuronal migration in the adult mammalian brain. Science 264:1145-1148.

Luskin MB (1993) Restricted proliferation and migration of postnatally generated neurons derived from the forebrain subventricular zone. Neuron 11:173-189.

Luskin MB, McDermott K (1994) Divergent lineages for oligodendrocytes and astrocytes originating in the neonatal forebrain subventricular zone. Glia 11:211-226.

Luskin MB, Pearlman AL, Sanes JR (1988) Cell lineage in the cerebral cortex of the mouse studied in vivo and in vitro with a recombinant retrovirus. Neuron 1:635-647.

Marin O, Rubenstein JLR (2001) A long, remarkable journey: tangential migration in the telencephalon. Nat Rev Neurosci 2:780-791.

Misson J-P, Edwards MA, Yamamoto M, Caviness Jr VS (1988) Identification of radial glial cells within the developing murine central nervous system: studies based upon a new immunohistochemical marker. Brain Res Dev Brain Res 44:95-108.

Nait-Oumesmar B, Decker L, Lachapelle F, Avellana-Adalid V, Bachelin C, Van Evercooren AB (1999) Progenitor cells of the adult mouse subventricular zone proliferate, migrate and differentiate into oligodendrocytes after demyelination. Eur J Neurosci 12:4357-4366.

Ono K, Yasui Y, Rutishauser U, Miller RH (1997) Focal ventricular origin and migration of oligodendrocyte precursors into the chick optic nerve. Neuron 19:283-292.

Parnavelas JG (1999) Glial cell lineages in the rat cerebral cortex. Exp Neurol 156:418-429.

Paterson JA, Privat A, Ling EA, Leblond CP (1973) Investigation of glial cells in semithin sections. III. Transformation of subependymal cells into glial cells as shown by radioautography after ${ }^{3} \mathrm{H}$-thymidine injection into the lateral ventricle of the brain of young rats. J Comp Neurol 149:83-102.

Price J, Thurlow L (1988) Cell lineage in the rat cerebral cortex: a study using retroviral-mediated gene transfer. Development 104:473-482.

Pringle NP, Richardson WD (1993) A singularity of PDGF alphareceptor expression in the dorsoventral axis of the neural tube may define the origin of the oligodendrocyte lineage. Development 117:525-533.

Pringle NP, Mudhar HS, Collarini EJ, Richardson WD (1992) PDGF receptors in the rat CNS: during late neurogenesis, PDGF alphareceptor expression appears to be restricted to glial cells of the oligodendrocyte lineage. Development 115:535-551.

Privat A, Leblond CP (1972) The subependymal layer and neighboring region in the brain of the young rat. J Comp Neurol 146:277-302.

Puelles L, Kuwana E, Puelles E, Bulfone A, Shimamura K, Keleher J, Smiga S, Rubenstein JLR (2000) Pallial and subpallial derivatives in the embryonic chick and mouse telencephalon, traced by the expression of the genes Dlx-2, Emx-1, Nkx-2.1, Pax-6, Tbr-1. J Comp Neurol 424:409-438

Rakic P (1974) Neurons in rhesus monkey visual cortex: systemic relation between time of origin and eventual disposition. Science 183:425-427.

Rothstein JD, Martin LJ, Levey AI, Dykes-Hoberg M, Jin L, Wu D, Nash N, Kuncl RW (1994) Localization of neuronal and glial glutamate transporters. Neuron 13:713-725.

Shibata T, Yamada K, Watanabe M, Ikenaka K, Wada K, Tanaka K, Inoue Y (1997) Glutamate transporter GLAST is expressed in the radial glia-astrocyte lineage of developing mouse spinal cord. J Neurosci 17:9212-9219.

Smart I (1961) The subependymal layer of the mouse brain and its cell production as shown by radioautography after thymidine- ${ }^{3} \mathrm{H}$ injection. J Comp Neurol 116:325-347.

Spassky N, Goujet-Zalc C, Parmantier E, Olivier C, Martinez S, Ivanova A, 
Ikenaka K, Macklin W, Cerruti I, Zalc B, Thomas JL (1998) Multiple restricted origin of oligodendrocytes. J Neurosci 18:8331-8343.

Staugaitis SM, Zerlin M, Hawkes R, Levine JM, Goldman JE (2001) Aldolase $\mathrm{C} /$ Zebrin II expression in the neonatal rat forebrain reveals cellular heterogeneity within the subventricular zone and early astrocytes differentiation. J Neurosci 21:6195-6205.

Stoykova A, Fritsch R, Walther C, Gruss P (1996) Forebrain patterning defects in Small eye mutant mice. Development 122:3453-3465.

Tekki-Kessaris N, Woodruff R, Hall AC, Gaffield W, Kimura S, Stiles CD, Rowitch DH, Richardson WD (2001) Hedgehog-dependent oligodendrocyte lineage specification in the telencephalon. Development 128:2545-2554.

Thompson RJ, Kynoch PAM, Willson VJC (1982) Cellular localization of aldolase C subunits in human brain. Brain Res 232:489-493.
Voigt T (1989) Development of glial cells in the cerebral wall of ferrets: direct tracing of their transformation from radial glia into astrocytes. J Comp Neurol 289:74-88.

Walther EU, Dichgans M, Maricich SM, Romito RR, Yang F, Dziennis S, Zackson S, Hawkes R, Herrup K (1998) Genomic sequences of aldolase C (Zebrin II) direct lacZ expression exclusively in nonneuronal cells of transgenic mice. Proc Natl Acad Sci USA 95:2615-2620.

Wilson SW, Rubenstein JLR (2000) Induction and dorsoventral patterning of the telencephalon. Neuron 28:641-651.

Woodruff R, Tekki-Kessaris N, Stiles CD, Rowitch DH, Richardson WD (2001) Oligodendrocyte development in the spinal cord and telencephalon: common themes and new perspectives. Int J Dev Neurosci 19: 379-385. 